\title{
ENCAPSULAÇÃO DE SUCO DE MARACUJÁ POR CO-CRISTALIZAÇÃO COM SACAROSE: CINÉTICA DE CRISTALIZAÇÃO E PROPRIEDADES FÍSICAS ${ }^{1}$
}

\author{
Zailer ASTOLFI-FILHO ${ }^{2,3}$, Ana C. SOUZA², Érika C. D. REIPERT ${ }^{4}$, Vânia R. N. TELIS ${ }^{2, *}$
}

\section{RESUMO}

O processo de co-cristalização consiste na concentração de um xarope de sacarose até a supersaturação, quando então é adicionado o material a ser encapsulado. A partir daí, a mistura é submetida a uma intensa agitação que induz à nucleação e à aglomeração do produto. Neste trabalho, a encapsulação de suco concentrado de maracujá em sacarose por co-cristalização foi avaliada, determinandose o efeito da fração de suco adicionada e do $\mathrm{pH}$ do suco sobre a umidade, solubilidade, densidade aparente e ângulo de repouso do produto final e acompanhando-se a cinética de cristalização em um reo-reator, constituído de um cristalizador acoplado a um reômetro rotacional de cilindros concêntricos, cujo cilindro interno foi substituído por um agitador. A cinética de co-cristalização foi representada por um modelo empírico ajustado aos dados obtidos. A co-cristalização foi acelerada em função do aumento do pH e da redução da porcentagem de suco. Os produtos co-cristalizados apresentaram menor umidade e maior solubilidade em baixas concentrações de suco. A densidade aparente e o ângulo de repouso foram similares aos da matriz encapsulante e situaram-se na faixa em que se encontram a maioria dos pós alimentícios.

Palavras-chave: microencapsulação, sucos de frutas, açúcar, pós, viscosidade aparente.

\section{SUMMARY}

ENCAPSULATION OF PASSION FRUIT JUICE BY CO-CRYSTALLIZATION WITH SUCROSE: CRYSTALLIZATION KINETICS AND PHYSICAL PROPERTIES. Co-crystallization is an encapsulation process where a second ingredient is incorporated in a porous conglomerate of sucrose microcrystals formed by spontaneous crystallization. The process is carried out by concentrating a sucrose syrup until supersaturation and, then, adding the core material, with the mixture being submitted to an intensive agitation that leads to nucleation and product agglomeration. In the present work, encapsulation of passion fruit concentrated juice by co-crystallization with sucrose was evaluated by determining the effects of added juice fraction and juice $\mathrm{pH}$ on moisture content, solubility, apparent density and repose angle of final product, as well as by following the co-crystallization kinetics in a rheo-reactor, constituted of a crystallizer adapted to a concentric cylinders rotational rheometer, in which an agitator substitutes for the internal cylinder. The cocrystallization kinetics was described by an empirical model fitted to experimental data and the crystallization rate was accelerated with increasing $\mathrm{pH}$ and decreasing added juice fraction. The co-crystallized products presented lower moisture content and higher solubility at lower juice fractions. Apparent density and repose angle were similar to those reported for the encapsulating matrix and were in the range of values reported for most of food powders.

Key-words: microencapsulation, fruit juice, sugar, food powders, apparent viscosity.

\section{1 - INTRODUÇÃO}

A microencapsulação é definida como a tecnologia de recobrir partículas ou pequenas gotas de material líquido ou gasoso, formando cápsulas em miniatura, as quais podem liberar seu conteúdo em taxas controladas e/ou sob condições específicas. Tais microcápsulas podem apresentar tamanho na faixa de frações de mícron até vários milímetros, possuindo diferentes formas, dependendo dos materiais e métodos utilizados em sua preparação. O material externo é denominado agente encapsulante, enquanto o ingrediente interno é o material ativo. Os carboidratos - entre eles a sacarose - são muito empregados como agentes encapsulantes de aromas devido a sua capacidade de absorver voláteis do ambiente ou retê-los fortemente durante o processo de secagem [17, 13]. Ingredientes alimentícios encapsulados em sacarose podem ser obtidos por co-cristalização. Nesse processo, a estrutura cristalina da sacarose é modificada, passando de um cristal perfeito para um conglomerado. Essa

\footnotetext{
${ }^{1}$ Recebido para publicação em 04/03/2005. Aceito para publicação em 09/09/2005 (001497).

${ }^{2} U N E S P$ - Universidade Estadual Paulista, Departamento de Engenharia e Tecnologia de Alimentos, 15054-000, São José do Rio Preto, SP.

${ }^{3}$ Açúcar Guarani S.A., Usina Cruz Alta, 15400-000, Olímpia, SP.

${ }^{4}$ UNICAMP - Universidade Estadual de Campinas, Departamento de Engenharia de Alimentos, 13083-862, Campinas, SP.

* A quem a correspondência deve ser enviada.
}

estrutura possui uma configuração porosa, que permite a adição de um segundo ingrediente. O produto apresenta baixa higroscopicidade e boas propriedades de fluidez e dispersão. Além disso, quando compostos sensíveis são aprisionados no interior dos aglomerados, ficam protegidos contra a oxidação $[2,4]$.

Durante a co-cristalização, a solução de sacarose é concentrada até o estado de supersaturação e mantida em temperatura elevada para evitar a cristalização. Uma quantidade pré-determinada de material ativo é então adicionada à solução concentrada de sacarose sob vigorosa agitação mecânica, promovendo a nucleação da mistura sacarose -material ativo. O aquecimento é interrompido e a mistura atinge a temperatura na qual se inicia a cristalização, sendo liberada uma quantidade substancial de calor devido à transição de fase, o que, por sua vez, contribui para a secagem do material. A agitação é continuada para promover a cristalização até a formação do produto aglomerado. O co-cristalizado é seco (se necessário) e peneirado para uniformizar a granulometria. Os materiais ativos encapsulados são incorporados nos interstícios entre os cristais $[11,1]$.

CHEN, VEIGA E RIZZUTO [6] citaram diversos produtos que podem ser encapsulados por co-cristalização com sacarose, tais como: sucos de frutas, óleos essenciais, chocolate, manteiga de amendoim e outros. BERISTAIN 
et al. [3] encapsularam óleo de casca de laranja por cocristalização e conduziram testes de armazenamento do produto resultante. Apesar de boa fluidez, o produto exigiu a adição de antioxidante devido à presença de óleo não encapsulado, resultante do excesso de material ativo em relação ao agente encapsulante. BHANDARI et al. [4] estudaram o processo de co-cristalização de mel com sacarose e obtiveram bons resultados quando a proporção de sacarose:mel foi superior a 85:15, sendo o produto resultante comparável ao mel original em termos de sabor e aroma. BERISTAIN et al. [2] empregaram a cocristalização para encapsular extrato de flores de hibisco (Hibiscus sabdarifa L.), o que resultou em um produto de boa dispersibilidade, solubilidade, homogeneidade e fluidez, além da retenção de todo o aroma, sabor e cor característicos do extrato vegetal. Esses autores observaram que existe um importante efeito do $\mathrm{pH}$ do extrato sobre as propriedades do produto resultante.

O suco de maracujá, devido a seus intensos sabor e aroma e alta acidez, tem sido descrito como um concentrado natural. Quando adoçado e diluído, fornece uma bebida de alta qualidade e muito apreciada. Para a aceitação mais geral do produto, a proporção de açúcar:suco deve ser maior que 45:100 e a taxa de diluição deve ser de 3 partes de água para 1 parte de suco [14]. O suco de maracujá é uma boa fonte de vitamina $\mathrm{C}$ e carotenóides (pró-vitamina A), podendo ser usado na preparação de geléias, recheios de tortas ou coberturas de bolos [12]. Considerando que o ácido ascórbico pode ser facilmente oxidado e degradado durante o armazenamento, a microencapsulação do suco de maracujá utilizando sacarose, a qual já é geralmente empregada em suas preparações, poderia ser uma alternativa para a melhor conservação de suas qualidades sensoriais e nutricionais. Soma-se a isso a vantagem de se obter um produto seco, com boas propriedades de solubilização, dispersão e fluidez.

O objetivo deste trabalho foi estudar a viabilidade de co-cristalização de suco de maracujá com sacarose, verificando a influência do $\mathrm{pH}$ do suco e da proporção suco: sacarose sobre algumas propriedades físicas - umidade, densidade aparente, ângulo de repouso, solubilidade - e sobre a cinética de cristalização do produto.

\section{2 - MATERIAL E MÉTODOS}

\section{1 - Matéria-prima}

Os xaropes foram preparados com sacarose comercial (açúcar refinado microcristalino) em água destilada. O suco de maracujá empregado foi do tipo concentrado congelado comercial $\left(64,5^{\circ} \mathrm{Brix}, \mathrm{pH}\right.$ original 2,96$)$. O ajuste do $\mathrm{pH}$ do suco antes da adição ao xarope foi feito em pH-metro digital (Digimed, modelo DM 20), usando solução de hidróxido de cálcio $4 \mathrm{~N}$.

\section{2 - Preparação das amostras para determinação das propriedades físicas}

Os xaropes de sacarose foram preparados em bateladas de cerca de $400 \mathrm{~g}$ e concentração inicial de $70^{\circ} \mathrm{Brix}$ em um recipiente aberto, de aço inoxidável, e aquecidos até $128^{\circ} \mathrm{C}$ em chapa aquecedora com agitação magnética (Fisatom, modelo 752 A). Em seguida, o suco de maracujá, já com o $\mathrm{pH}$ corrigido, foi adicionado ao xarope e deu-se início imediato à agitação, que permaneceu constante até a formação de um material sólido particulado. Nessa etapa foi usado um agitador mecânico portátil, adaptado a partir de uma retificadeira (Maquita, modelo 906, freqüência 50 a $60 \mathrm{~Hz}$ e potência $240 \mathrm{~W}$ ), cujo disco de polimento foi substituído por um rotor de pás metálicas.

Terminada a agitação, o material foi submetido à secagem em estufa com circulação de ar a $50^{\circ} \mathrm{C}$ (Marconi, modelo MA 037) por 12 horas, seguida de moagem e peneiramento em malha 20 . O produto foi armazenado em frascos de vidro hermeticamente fechados e guardado em geladeira por cerca de duas semanas até a realização de todas as análises.

\section{3 - Umidade}

A umidade do produto co-cristalizado foi determinada em triplicata, pelo método gravimétrico, com secagem em estufa a vácuo (Marconi, modelo MA 030) a $60^{\circ} \mathrm{C}$ por 48 horas. As pesagens foram feitas em balança analítica (Chyo, modelo JK 200).

\section{4 - Densidade aparente}

A densidade aparente foi avaliada em triplicata, medindo-se o volume ocupado por aproximadamente $30 \mathrm{~g}$ de pó depositado em uma proveta graduada de $250 \mathrm{~mL}$. Foram aplicadas leves batidas da proveta contra uma superfície plana, até que o volume ocupado pelo pó atingisse um valor constante, o qual era utilizado no cálculo da densidade [3].

\section{5 - Ângulo de repouso}

Medidas do ângulo de repouso estático foram utilizadas como indicação da capacidade de escoamento ou fluidez do produto. O material foi despejado vagarosamente de uma altura fixa através de um funil de vidro colocado em um suporte, sendo coletado em uma placa de Petri. A partir do raio da placa de Petri e da altura do cone formado pelo pó, foi possível a determinação do ângulo de repouso [4]. As medidas foram feitas em triplicata.

\section{6 - Solubilidade}

Foi determinada em triplicata, medindo-se o tempo necessário para a completa dissolução de $10 \mathrm{~g}$ do produto em $100 \mathrm{~mL}$ de água destilada a $25^{\circ} \mathrm{C}$. A mistura foi agitada manualmente com um bastão de vidro e o tempo, medido em um cronômetro, correspondeu ao momento em que não mais foram observadas partículas sólidas [3]. 


\section{7 - Cinética de cristalização}

Para o estudo da cinética de cristalização, utilizou-se um cristalizador montado na base de um reômetro rotacional de cilindros concêntricos, (MLW, modelo Rheotest 2.1, Alemanha). Um agitador do tipo âncora foi acoplado ao eixo originalmente destinado ao cilindro interno. O vaso cristalizador foi encamisado e conectado a um banho termostático.

Os xaropes de sacarose foram preparados em bateladas de cerca de $700 \mathrm{~g}$ e concentração inicial de $77^{\circ} \mathrm{Brix}$ em um recipiente aberto, de aço inoxidável, e aquecidos até $116^{\circ} \mathrm{C}$ em chapa aquecedora com agitação magnética (Fisatom, modelo 752 A). Em seguida, o suco de maracujá, já com o pH corrigido, foi adicionado ao xarope e, imediatamente, $340 \mathrm{~mL}$ dessa mistura foram colocados no cristalizador, sendo acionado o reômetro, cuja velocidade de rotação foi mantida constante e igual a $81 \mathrm{rpm}$. Durante o processo, leituras de torque foram periodicamente registradas.

Os experimentos no reo-cristalizador foram conduzidos de acordo com um planejamento fatorial $2^{2}$, com o pH do suco variando de 3,5 a 4,5 e a proporção de suco adicionada ao xarope de sacarose variando de 10 a $15 \%$ em peso. Esses ensaios foram realizados em triplicata.

\subsection{1 - Cálculo da viscosidade aparente ao longo da cristalização}

O método descrito a seguir é baseado na analogia de Couette e permite a análise quantitativa dos dados de torque e velocidade rotacional, de forma a extrair dados absolutos de viscosidade $(\eta)$ versus taxa de deformação $(\dot{\gamma})$ usando geometrias não convencionais [11]. Tal analogia consiste primeiramente em determinar o raio, $\mathrm{R}_{\mathrm{i}}$, de um cilindro interno de Couette equivalente, o qual teria a mesma altura, L, do agitador e que, para uma velocidade rotacional constante, $\mathrm{N}$, causaria o mesmo torque, $\mathrm{T}$, no vaso cilíndrico de raio Re. Resolvendo as equações de transferência de quantidade de movimento para esta geometria virtual de Couette em regime permanente, com escoamento laminar e isotérmico de um fluido que obedeça à lei da potência, em que a viscosidade aparente (

$$
\eta_{a p}=K(\gamma)^{n-1}
$$

em que k e n são os índices de consistência e de comportamento do fluido, respectivamente, obtém-se a seguinte Equação (2) para Ri:

$$
\mathrm{R}_{\mathrm{i}}=\frac{\mathrm{R}_{\mathrm{e}}}{\left[1+\left(\frac{4 \pi \mathrm{N}}{\mathrm{n}}\right)\left(\frac{2 \pi \mathrm{KLR}_{\mathrm{e}}^{2}}{\mathrm{~T}}\right)^{1 / \mathrm{n}}\right]^{\mathrm{n} / 2}}
$$

Para um determinado par (N, T), CHOPLIN, CHAVEZMONTES e SCHAER [11] observaram que $R_{i}$ depende apenas ligeiramente do índice n. Dessa forma, é possível determinar $\mathrm{R}_{\mathrm{i}}$ utilizando um fluido newtoniano de viscosidade conhecida $(n=1)$. Uma vez que $R_{i}$ equivalente tenha sido determinado, podem-se determinar os perfis de tensão de cisalhamento e taxa de deformação, a partir dos quais se deduz a viscosidade em uma posição particular do espaço anular virtual onde se encontra o fluido. A tensão de cisalhamento é dada pela Equação (3):

$$
\sigma=\frac{T}{2 \pi \mathrm{Lr}^{2}}
$$

e a taxa de deformação, para um fluido lei da potência, pela Equação (4):

$$
\dot{\gamma}=\left[\frac{\frac{4 \pi}{n}\left(\frac{R_{i}}{r}\right)^{2 / n}}{1-\left(\frac{R_{i}}{R_{e}}\right)^{2 / n}}\right] N
$$

Mesmo para um grande espaço virtual entre os cilindros, verifica-se que existe uma posição específica $r=r^{*}$ em que o termo entre colchetes na Equação (4) é essencialmente independente do índice $\mathrm{n}$, isto é, da reologia do fluido. $\mathrm{O}$ valor do raio equivalente, $\mathrm{r}^{*}$, pode ser calculado usando a Equação (4) para n=1 e avaliando a tensão de cisalhamento e a taxa de deformação nesse valor específico $r=r^{*}$. Ou seja (Equações 5 e 6):

$$
\begin{gathered}
\sigma_{\mathrm{r}^{*}}=\frac{\mathrm{T}}{2 \pi \mathrm{L}\left(\mathrm{r}^{*}\right)^{2}} \\
\dot{\gamma}_{r^{*}}=\frac{\frac{4 \pi N}{n}\left(\frac{R_{i}}{\mathrm{r}^{*}}\right)^{2 / n}}{1-\left(\frac{R_{i}}{\mathrm{r}_{\mathrm{e}}}\right)^{2}}
\end{gathered}
$$

A viscosidade aparente é então obtida da razão entre $\sigma_{r^{*}}$ e $\dot{\gamma}_{r^{*}}$ correspondendo à taxa de deformação $\dot{\gamma}_{\mathrm{r}^{*}}$. CHOPLIN, CHAVEZ-MONTES e SCHAER [8] testaram esse método para vários agitadores e para diversos sistemas reologicamente complexos, como maionese, molhos para salada e formulações de sorvetes. Seus resultados mostraram boa concordância (erro máximo de 5\%) com os obtidos em geometrias convencionais em uma grande faixa de taxas de deformação.

\subsection{2 - Determinação do raio equivalente do agitador}

O raio equivalente $\left(r^{*}\right)$ do agitador utilizado neste trabalho foi determinado usando etilenoglicol e glicerina a $30^{\circ} \mathrm{C}$, cujas viscosidades foram tomadas, respectivamente, como $11,61 \mathrm{mPa} . \mathrm{s}$ e $16,86 \mathrm{mPa} . \mathrm{s}$ [16]. 


\section{8 - Planejamento experimental e tratamento estatístico}

Para a determinação das propriedades físicas, as amostras foram preparadas de acordo com um planejamento fatorial $2^{2}$, com duplicata do ponto central, com o pH do suco variando de 3,5 a 4,5 e a proporção de suco adicionada ao xarope de sacarose variando de 10 a $15 \%$ em peso [5]. Nos ensaios de cinética de cristalização, não foi considerado o ponto central.

O tratamento estatístico dos resultados foi feito por meio de análise de variância usando o módulo Experimental Design do software Statistica v. 5.0 [18], estabelecendo-se um intervalo de confiança de $95 \%$.

\section{3 - RESULTADOS E DISCUSSÃo}

\section{1 - Propriedades físicas}

Os resultados obtidos para as propriedades físicas do produto co-cristalizado são apresentados na Tabela 1. Os efeitos do pH do suco e da proporção suco: sacarose sobre as mesmas foi avaliado estatisticamente de acordo com o item 2.8. Modelos empíricos, de acordo com a Equação (7) onde "Resposta" corresponde à umidade, densidade aparente, ângulo de repouso ou tempo de solubilização, foram ajustados aos resultados experimentais, sendo os parâmetros $\beta_{i}$ s os coeficientes de regressão do modelo ajustado. Os valores destes parâmetros, bem como o respectivo nível de significância (p), são apresentados na Tabela 2.

$$
\text { Resposta }=\beta_{0}+\beta_{1}(\% \text { Suco })+\beta_{2}(\mathrm{pH})+\beta_{12}(\% \text { Suco })(\mathrm{pH})
$$

Para a umidade, a análise de variância indicou que apenas o efeito da proporção de suco foi significativo em nível de $5 \%$ (Tabela 2). Os dados da Tabela 1 mostram que as maiores umidades ocorreram na região de maiores conteúdos de suco, sendo possível observar também um ligeiro aumento da umidade na região de maiores pHs. Os resultados apresentados por BERINSTAIN et al. [2,3] e por BHANDARI et al. [4] também indicaram maiores umidades para co-cristalizados obtidos com maiores porcentagens do material encapsulado.

Os conteúdos de umidade obtidos para o co-cristalizado de suco de maracujá foram mais altos que os valores encontrados na prática para o açúcar microcristalino comercial - o qual constitui a matriz encapsulante do produto estudado - que varia de 0,1 a 0,3\% [7]. Entretanto, um pequeno ajuste nas condições de secagem do produto final deve ser suficiente para deixá-lo de acordo com o padrão comercial (máximo de 0,3\% em base úmida [9]).

Os resultados apresentados na Tabela 1 mostram que há uma tendência de aumento da densidade aparente em baixas concentrações de suco, o que pode indicar que menores frações de suco levam a um produto de menor porosidade. É possível que, na presença de menor quantidade do material encapsulado, uma maior fração de sacarose tenha cristalizado na sua forma habitual, formando partículas mais compactadas e de faces mais planas. A análise de variância indicou efeito linear significativo para a porcentagem de suco de maracujá adicionado, enquanto o $\mathrm{pH}$ não apresentou efeito sobre a resposta considerada (Tabela 2).

É importante, ainda, salientar que o aumento da densidade não pode ser atribuído à ocorrência de maiores umidades, apesar de que o aumento da umidade, especialmente em pós contendo compostos cristalinos solúveis - tais como açúcares ou sais - pode resultar na liquefação do material e, conseqüentemente, em um aumento de sua densidade aparente [15]. Os resultados mostram que a proporção de suco adicionada provocou efeito contrário na umidade e na densidade aparente dos produtos. De modo geral, os valores de densidade aparente dos produtos co-cristalizados com suco de maracujá (entre 0,638 e 0,769 g/ $\mathrm{cm}^{3}$ ) encontram-se na faixa correspondente à maioria dos pós alimentícios, que

TABELA 1 - Propriedades físicas em função do pH e da proporção de suco adicionado

\begin{tabular}{cccccc}
\hline \% Suco & $\mathbf{p H}$ & Umidade (\% b.u.) & Densidade aparente $\mathbf{( g / \mathbf { c m } ^ { 3 } )}$ & Ângulo de repouso (graus) & Tempo de solubilização (s) \\
\hline \multirow{2}{*}{15} & 4,5 & 1,02 & 0,652 & 31,60 & 60,00 \\
& 3,5 & 0,95 & 0,638 & 28,45 & 40,31 \\
\multirow{2}{*}{10} & 4,5 & 0,27 & 0,750 & 32,62 & 36,71 \\
& 3,5 & 0,21 & 0,769 & 35,56 & 40,40 \\
12,5 & 4,0 & 0,31 & 0,750 & 33,62 & 43,23 \\
& 4,0 & 0,32 & 0,750 & 34,60 & 45,12 \\
\hline
\end{tabular}

TABELA 2 - Coeficientes de regressão para a Equação (7) ajustada a cada uma das propriedades físicas. Os números entre parênteses indicam o nível de significância $(p)$ do fator

\begin{tabular}{|c|c|c|c|c|}
\hline Fator & Umidade ( $\%$ b.u.) & Densidade aparente $\left(\mathrm{g} / \mathrm{cm}^{3}\right)$ & Ângulo de repouso (graus) & Tempo de solubilização (s) \\
\hline Interseção $\left(\beta_{0}\right)$ & $-1,069(0,185)$ & $1,014(0,008)$ & $42,28(0,029)$ & $217,1(0,081)$ \\
\hline$\%$ Suco $\left(\beta_{1}\right)$ & $0,149(0,033)$ & $-0,023(0,041)$ & $-0,813(0,170)$ & $-16,38(0,009)$ \\
\hline $\mathrm{pH}\left(\beta_{2}\right)$ & $0,065(0,765)$ & $-0,003(0,945)$ & $0,105(0,966)$ & $-50,45(0,009)$ \\
\hline$\%$ Suco x pH $\left(\beta_{12}\right)$ & - & - & - & $4,676(0,007)$ \\
\hline
\end{tabular}


vai de 0,30 a $0,80 \mathrm{~g} / \mathrm{cm}^{3}$ [ 15]. BERISTAIN et al. [2] apresentaram resultados da densidade aparente do co-cristalizado com extrato de hibisco entre 0,40 e 0,60 g/cm3, valores pouco inferiores aos obtidos neste trabalho.

Para o ângulo de repouso, foram obtidos valores próximos entre si, independentemente das condições de co-cristalização (Tabela 1) e a análise estatística não indicou nenhum efeito significativo das variáveis estudadas (Tabela 2). Existe apenas uma tendência de diminuição do ângulo de repouso em função do aumento da fração de suco de maracujá adicionado.

A medida do ângulo de repouso é um método simples de caracterização do comportamento de pós ou grânulos durante o escoamento. Os pós que exibem ângulos de repouso menores que $45^{\circ}$ geralmente apresentam a propriedade de escoamento livre, enquanto ângulos acima de $50^{\circ}$ indicam coesividade ou problemas de escoamento [4]. Todas as amostras avaliadas apresentaram ângulos em torno de $30^{\circ}$, o que pode ser considerado tecnicamente adequado. Para o açúcar refinado comercial (matriz encapsulante), o ângulo de repouso encontra-se na faixa de $30^{\circ}$ a $37^{\circ}$, com um valor médio de $34,7^{\circ} \pm 1,4^{\circ}[7]$.

Considerando o tempo de solubilização dos produtos co-cristalizados com suco de maracujá, tanto a proporção de suco adicionada quanto o pH apresentaram efeitos significativos $(p<0,05)$, bem como a interação entre estas variáveis. As amostras de menor solubilidade correspondem às maiores proporções de suco e aos maiores pHs. Esse efeito poderia ser atribuído ao fato de que o material encapsulante, isto é, a sacarose microcristalina, apresenta solubilidade bastante alta. Segundo GARCIA et al. [9], o açúcar refinado é usado na preparação de misturas sólidas de dissolução instantânea. Dessa forma, a adição de uma determinada proporção de suco de frutas estaria retardando a solubilização. BERINSTAIN et al. [2] não observou efeitos significativos de $\mathrm{pH}$ e porcentagem de extrato adicionado sobre a solubilidade do açúcar co-cristalizado com concentrado de hibisco, mas considerou o produto, cujo tempo de solubilização situou-se 28,3 e 35 s, como tendo excelentes propriedades de reconstituição. Os tempos de solubilização apresentados na Tabela 1 encontram-se entre 36,7 e 60,0 s, um pouco superiores aos relatados por BERINSTAIN et al. [2], mas ainda característicos de produtos de boa solubilidade.

\section{2 - Cinética de co-cristalização}

\subsection{1 - Raio equivalente $\left(r^{*}\right)$ do agitador}

Os resultados da calibração do agitador acoplado ao reômetro com etilenoglicol e glicerina para obtenção do raio equivalente $\left(\mathrm{r}^{*}\right)$ são apresentados na Tabela $3 \mathrm{e}$, como pode ser observado, o valor a ser utilizado para a obtenção das taxas de deformação e tensões de cisalhamento, independentemente da velocidade de rotação do agitador, deve ser igual a $0,29 \mathrm{~m}$.

\subsection{2 - Co-cristalização do suco de maracujá}

A Figura 1 mostra a viscosidade aparente em função do tempo para os ensaios de co-cristalização realizados em diferentes condições de $\mathrm{pH}$ do suco e proporção suco:sacarose. Nessa figura, a ordenada representa a relação $\eta_{\mathrm{ap}} / \eta_{0}$, isto é, a razão entre a viscosidade aparente do sistema ao longo do tempo de cristalização e a viscosidade aparente inicial do sistema, sendo esta obtida a partir da leitura de torque realizada no início do processo (tempo 0).

Comparando-se as três curvas experimentais obtidas para cada condição de co-cristalização observa-se, de modo geral que, no início do processo, existe uma grande coincidência das mesmas ao longo do tempo. Entretanto, à medida que o processo avança, as viscosidades observadas passam a diferir entre si. É possível que essa discordância deva-se às condições atmosféricas, uma vez que o sistema em questão é altamente higroscópico. Mesmo durante os ensaios de co-cristalização com agitação manual, já foi observado em nosso laboratório que o tempo necessário para completar o processo varia muito em função da umidade relativa do ambiente. No entanto, mesmo com essa dificuldade experimental, é possível tirar

TABELA 3 - Calibração do agitador acoplado ao reômetro

\begin{tabular}{|c|c|c|c|c|c|}
\hline N (rpm) & Torque (N.m) & $R_{i}\left(10^{-2} \mathrm{~m}\right)$ & $\Omega(\mathrm{rad} / \mathrm{s})$ & $\dot{\gamma}\left(s^{-1}\right)$ & $r^{*}(m)$ \\
\hline \multicolumn{6}{|c|}{ Etilenoglicol } \\
\hline 40,5 & 0,8 & 3,73 & 4,24 & 797 & 0,290 \\
\hline 45,0 & 1,0 & 3,75 & 4,71 & 1180 & 0,290 \\
\hline 67,5 & 2,4 & 3,74 & 7,07 & 2653 & 0,290 \\
\hline 81,0 & 3,8 & 3,74 & 8,48 & 3184 & 0,290 \\
\hline 121,5 & 8,0 & 3,75 & 12,70 & 9544 & 0,290 \\
\hline 135,0 & 10,4 & 3,75 & 14,10 & 10605 & 0,290 \\
\hline 243,0 & 33,0 & 3,75 & 25,40 & 47703 & 0,290 \\
\hline \multicolumn{6}{|c|}{ Glicerina } \\
\hline 67,5 & 1,8 & 3,74 & 7,07 & 2211 & 0,285 \\
\hline 81,0 & 2,5 & 3,74 & 8,48 & 2895 & 0,290 \\
\hline 121,5 & 5,8 & 3,74 & 12,70 & 6821 & 0,290 \\
\hline 135,0 & 6,9 & 3,74 & 14,10 & 8838 & 0,290 \\
\hline 243,0 & 18,2 & 3,75 & 25,40 & 23853 & 0,290 \\
\hline
\end{tabular}



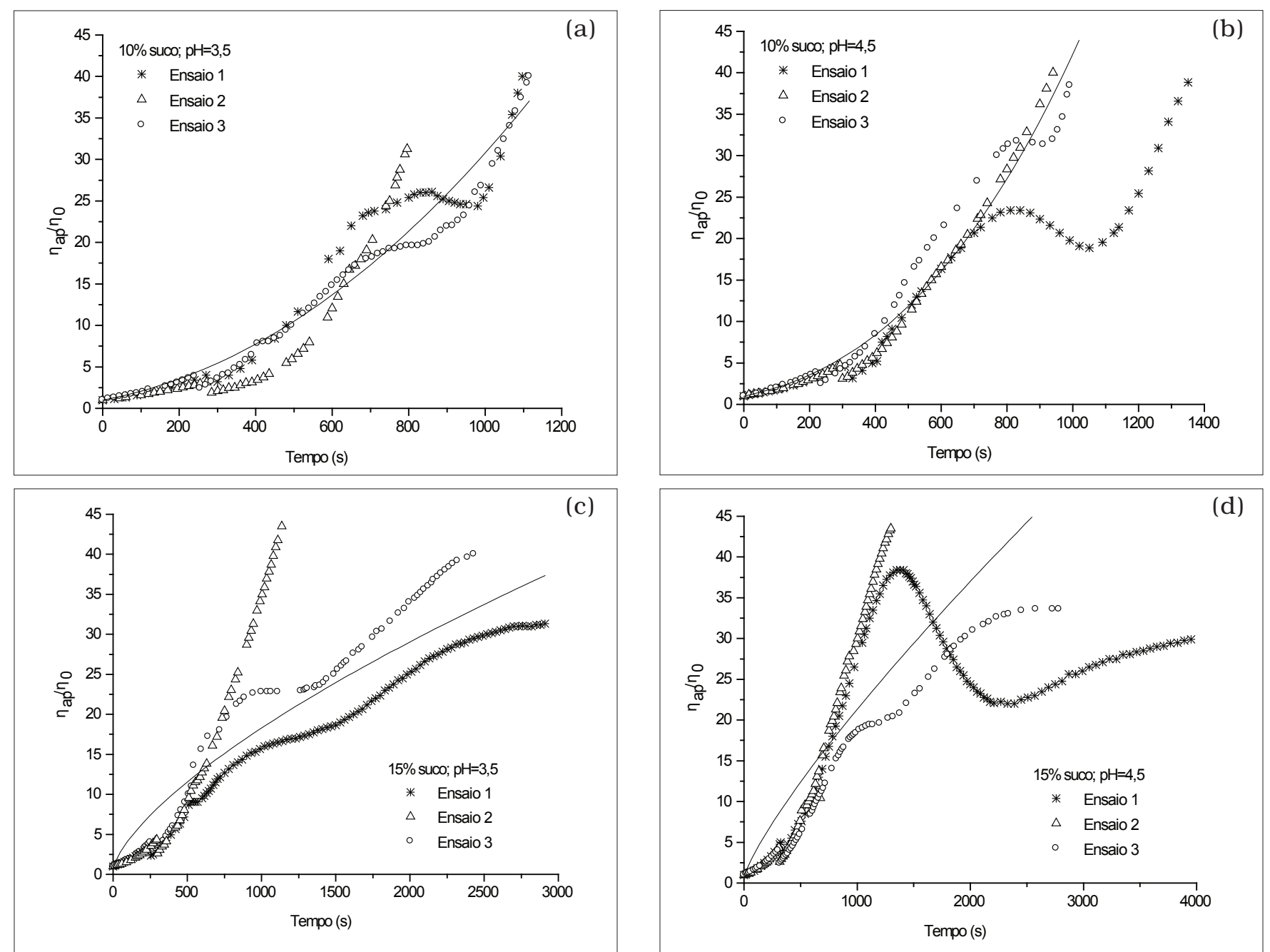

FIGURA 1 - Viscosidade aparente ao longo do tempo para co-cristalização de suco de maracujá em diferentes condições: (a) 10\% de suco e pH 3,5; (b) $10 \%$ de suco e pH 4,5; (c) $15 \%$ de suco e pH 3,5; (d) $15 \%$ de suco e pH 4,5

conclusões a partir da análise da Figura 1. Tomando-se como base o total de dados obtidos nas três repetições de cada condição de co-cristalização, ajustou-se um modelo empírico para representar a cinética de cristalização em termos da variação de viscosidade aparente do sistema em função do tempo. O modelo dado pela Equação (8) e representado pelas linhas sólidas na Figura 1 é similar ao modelo apresentado por KIM, LEE e AHN [10] para representar a viscosidade aparente ao longo do processo de cristalização de um sistema originalmente no estado vítreo, no qual o tempo de cristalização foi implicitamente representado pela fração volumétrica de fase cristalina que aumenta ao longo do tempo de cristalização.

$$
\frac{\eta_{\mathrm{ap}}}{\eta_{0}}=(1+\alpha \mathrm{t})^{\beta}
$$

Na Equação (8), $\eta_{\mathrm{ap}}$ é a viscosidade aparente do sistema constituído de solução supersaturada e microcristais, $\eta_{0}$ é a viscosidade inicial do sistema, que corresponde à solução supersaturada de sacarose, já misturada ao suco concentrado, t é o tempo de cristalização e $\alpha$ e $\beta$ são parâmetros empíricos. KIM, LEE e AHN [10] afirmam que as constantes de ajuste estão relacionadas à forma e orientação dos cristais dispersos no sistema vítreo. No caso da co-cristalização, observou-se que as constantes $\alpha$ e $\beta$ podem ser relacionadas às condições de co-cristalização. A Tabela 4 apresenta um resumo dos parâmetros de ajuste da Equação (8) para cada condição de co-cristalização.

Observa-se na Tabela 4 que os ensaios com menor porcentagem de suco tendem a apresentar menores valores de $\alpha$ e maiores valores de $\beta$. Em relação ao efeito do $\mathrm{pH}$,

TABELA 4 - Parâmetros da Equação (8) em função das condições de co-cristalização

\begin{tabular}{cccc}
\hline \% Suco & $\mathrm{pH}$ & $\boldsymbol{\alpha} \times 10^{\mathbf{2}}\left(\mathbf{s}^{-1}\right)$ & $\boldsymbol{\beta}$ \\
\hline 10 & 3,5 & $0,42 \pm 0,06$ & $2,08 \pm 0,15$ \\
& 4,5 & $0,30 \pm 0,03$ & $2,71 \pm 0,15$ \\
\multirow{2}{*}{15} & 3,5 & $7,33 \pm 1,96$ & $0,68 \pm 0,04$ \\
& 4,5 & $4,27 \pm 1,16$ & $0,81 \pm 0,05$ \\
\hline
\end{tabular}


verificou-se o oposto: as amostras com menor $\mathrm{pH}$ apresentaram maiores valores de $\alpha$ e menores valores de $\beta$.

Valores de $\beta$ maiores que 1 , fazem com que a velocidade de cristalização aumente ao longo do tempo, como se observa nas curvas correspondentes à menor porcentagem de suco. Por outro lado, se $\beta$ é menor que 1 , a velocidade de cristalização diminui com o tempo, como ocorre para os sistemas com $15 \%$ de suco. Além disso, o valor de $\alpha$ se reflete na posição relativa das curvas com diferentes $\mathrm{pHs}$ e com a mesma porcentagem de suco: as curvas obtidas para o $\mathrm{pH}$ de 4,5 ficam acima daquelas correspondentes ao $\mathrm{pH}$ de 3,5. Nesse caso, os menores valores de $\alpha$ são compensados pelos maiores valores de $\beta$.

Conclui-se que a co-cristalização é favorecida pela menor fração de suco adicionada e pelo maior $\mathrm{pH}$ do suco.

\section{4 - CONCLUSÕES}

Considerando os efeitos da fração de suco adicionada e de seu pH sobre as propriedades físicas dos produtos co-cristalizados e sobre a cinética de co-cristalização, podemos concluir que:

- a porcentagem de suco adicionada deve ser a mais baixa possível, desde que garanta a funcionalidade do produto, uma vez que, à medida que aumenta a fração de suco adicionada, aumenta o tempo de cristalização, aumenta a umidade final do produto e diminui a sua solubilidade;

- $\quad \mathrm{O}$ pH não apresentou efeito significativo sobre a umidade, porém a velocidade de cristalização foi maior para o $\mathrm{pH}$ de 4,5;

- A densidade aparente e o ângulo de repouso não foram afetados significativamente pelo $\mathrm{pH}$ e pela fração de suco adicionada, porém apresentaram valores satisfatórios e similares à maioria dos pós alimentícios, incluindo a matriz encapsulante;

- É possível usar o reo-reator para determinar a cinética de co-cristalização; entretanto, o sistema utilizado e a metodologia ainda precisam ser aperfeiçoados para eliminar a influência de fatores externos ao processo.

\section{5 - REFERÊNCIAS BIBLIOGRÁFICAS}

[1] AWAD, A.; CHEN, A.C. A new generation of sucrose products made by cocrystallization. Food Technology, v. 47, n. 1, p. 146-148, 1993.

[2] BERISTAIN, C.I.; MENDOZA, R.E.; GARCIA, H.S.; VAZQUEZ, A. Cocrystallization of jamaica (Hibiscus sabdarifa L.) granules. Lebensmittel-Wissenschaft und-Technolgie, v. 27, n. 4, p. 347-349, 1994.

[3] BERISTAIN, C.I.; VAZQUEZ, A.; GARCIA, H.S.; VERNON-CARTER, E.J. Encapsulation of orange peel oil by co-crystallization. Lebensmittel-Wissenschaft und-Technolgie, v. 29, n. 7, p. 645-647, 1996.

[4] BHANDARI, B.R.; DATTA, N.; D'ARCY, B.R.; RINTOUL, G.B. Co-crystallization of honey with sucrose. Lebensmittel-Wissenschaft und-Technolgie, v. 31 , n. 2, p.
138-142, 1998.

[5] BOX, G.E.P.; HUNTER, W.G.; HUNTER, J.S. Statistics for Experimenters: an Introduction to Design, Data Analysis and Model Building. New York: John Wiley, 1978.

[6] CHEN, A.C.; VEIGA, M.F.; RIZZUTO, A.B. Co-crystallization: an encapsulation process. Food Technology, v. 42 , n. 11 , p. 87-90, 1988.

[7] CHEN, J.C.P. Cane Sugar Handbook. Houston: John Wiley, 1999.

[8] CHOPLIN, L.; CHAVEZ-MONTES, B.E.; SCHAER, E. Rheo-reactor for studying food processes: specific cases of foaming and freezing. In: WELTI-CHANES, J.; BARBOSA-CANOVAS, G.V.; AGUILERA, J.M. (Ed.) Engineering and Food for the $21^{\text {st }}$ Century. Boca Raton: CRC Press, 2002. Cap. 27, p. 447-462.

[9] Garcia, E.E.C.; Ardito, E.F.G.; Grosso, C.R.F.; Leite, R.S.S.F.; Savitci, L.A.; Gasparino Filho, J.; Pinto Neto, M. O uso de polietileno de baixa densidade (PEBD) para embalagem de até $5 \mathrm{Kg}$ de açúcar cristal e refinado. CETEA/ITAL, setembro, 8 p., 1992.

[10] KIM, K.D.; LEE, S.H.; AHN, H.K. Observation of nucleation effect on crystallization in lithium aluminosilicate glass by viscosity measurement, Journal of Non-Crystalline Solids, v. 336, n. 3, p. 195-201, 2004.

[11] KING, A.H. Encapsulation of food ingredients. In: RISCH, S. J.; REINECCIUS, G. A. (Ed.) Encapsulation and Controlled Release of Food Ingredients. Washington DC: ACS Symposium Series, 1995. p. 26-39.

[12] KNIGHT JR., R.J.; SAULS, J.W. The passion fruit. Fact Sheet HS-60, Horticultural Sciences Department, Florida Cooperative Extension Service, Institute of Food and Agricultural Sciences, University of Florida, 1994. Disponível em: < http://edis.ifas.ufl.edu/MG328>. Acesso em: 18 jan. 2005.

[13] NORI, M.A. Ingredientes e aditivos microencapsulados na indústria de alimentos. Engenharia de Alimentos, setembro, p. 33-35, 1996.

[14] OKOTH, M.W.; KAAHWA, A.R.; IMUNGI, J.K. The effect of homogenization, stabilizer and amylase on cloudiness of passion fruit juice. Food Control, v. 11, n. 4, p. 305-311, 2000.

[15] PELEG, M. Physical characteristics of food powders. In: PELEG, M.; BAGLEY, E.B. (Ed.) Physical Properties of Foods. Westport: AVI Publishing Co., 1983. p. 293-323.

[16] PERRY, R.; CHILTON, C. Manual de Engenharia Química, 5 ${ }^{a}$ ed. Rio de Janeiro: Guanabara Dois, 1986.

[17] SHAHIDI, F.; HAN, X.Q. Encapsulation of food ingredients. Critical Reviews in Food Science and Nutrition, v. 33, n. 6, p. 501-547, 1993.

[18] StatSoft, Inc. STATISTICA for Windows [Computer program manual]. StatSoft, Inc.: Tulsa, USA. 1995.

\section{6 - AGRADECIMENTOS}

Os autores agradecem à FAPESP (Processos 03/058026, 02/06838-1 e 01/03148-1) pelo auxílio financeiro e à empresa Açúcar Guarani S.A. pela doação de sacarose microcristalina. 